\title{
A New Flap-type Wave Maker without Water on Back Side
}

\author{
Kaname Taniguchi*, Member \\ Hironao Kasai**, Member
}

\begin{abstract}
Summary
A new type wave maker without water on back side was developed and installed in the Seakeeping and Manoeuvring Basin of Nagasaki Technical Institute, M.H.I. The design theory of this new type wave maker, construction device of the gap of the flap ends assuring water tightness as well as no disturbance on wave making and the system design are described, followed by a description of the new type wave maker of the Basin.
\end{abstract}

\section{Introduction}

The flap-type wave maker has been used widely in various experimental tanks, owing to its simplicity and reliability. The theory of wave making by a flap is well established and the agreement with the experiment is also confirmed.1) However, the flap-type wave maker generates waves on the both sides of the flap and the waves generated on the back side have to be absorbed by a proper device. Thus, considerable space and driving power are needed which are not utilized effectively to generate waves. On the other hand, the plunger-type and the pneumatic-type wave maker generate waves on one side only, so they do not have such disadvantages as the flap-type does. But the plunger-type requires a considerable space above the water surface and this makes it almost impossible to adopt such design that the towing carriage can run over the wave maker. As for the pneumatic-type wave maker the design theory is not fully established, and the shape of waves generated is not so good as the ones generated by the flap-type or the plunger-type wave maker. Therefore, if the disadvantage of the flap-type could be eliminated, we should be able to expect the most desirable wave maker.

With this view, the authors have been engaged in the improvement of the flap-type wave maker and developed a new flap-type wave maker without water on back side.

In the course of development, there were two difficult problems to be solved, the one is the construction design of the ends of the flap which assures the water tightness and also causes no disturbance on wave making, and the other is the lack of design theory which can be applied to this new type of wave maker. This new type wave maker is installed in the new Seakeeping and Manoeuvring Basin of Nagasaki Technical Institute, M.H.I. The wave maker has two groups of flaps; the one for the shorter side of the Basin is 30 meters in breadth, the other for the longer side is 120 meters in breadth, capable of generating waves up to 60 centimeters in height and 10 meters in length.

In the following, the design theory, the treatment of the flap ends and the system design of this new type wave maker are reported and also the description of the new wave maker of the Seakeeping and Manoeuvring Basin of Nagasaki Technical Institute is given as an example of the practical application.

\footnotetext{
* Director and Manager of Nagasaki Technical Institute, M.H.I.

** Seakeeping Research Laboratory, Nagasaki Technical Institute, M.H.I.
} 


\section{Design Theory}

As reported by one of the present authors, the velocity potential of the motion of water generated by the motion of the flap hinged at its bottom end is given as follows ${ }^{1)}$ :

$$
\begin{aligned}
\phi= & \frac{2 a \omega}{b}\left\{\frac{\cosh k_{0} H\left(\frac{b_{1} \omega^{2}}{g}-1\right)+\cosh k_{0}\left(H-b_{1}\right)}{k_{0} 3\left(H+\frac{g}{\omega^{2}} \sinh ^{2} k_{0} H\right)} \sin \left(\omega t-k_{0} x\right) \cosh k_{0}(y+H)\right. \\
& -\cos \omega t \sum_{m=1}^{\infty} \frac{\cos k_{m} H\left(-\frac{b_{1} \omega^{2}}{g}-1\right)+\cos k_{m}\left(H-b_{1}\right)}{k_{m}\left(H-\frac{g}{\omega^{2}} \sin ^{2} k_{m} H\right)} e^{-k_{m} x} \cos k_{m}(y+H)
\end{aligned}
$$

where $k_{0}$ and $k_{m}$ are the roots of the following equations.

$$
\begin{aligned}
k_{0} \tanh k_{0} H & =\frac{\omega^{2}}{g} \\
-k_{m} \tan k_{m} H & =\frac{\omega^{2}}{g}
\end{aligned}
$$

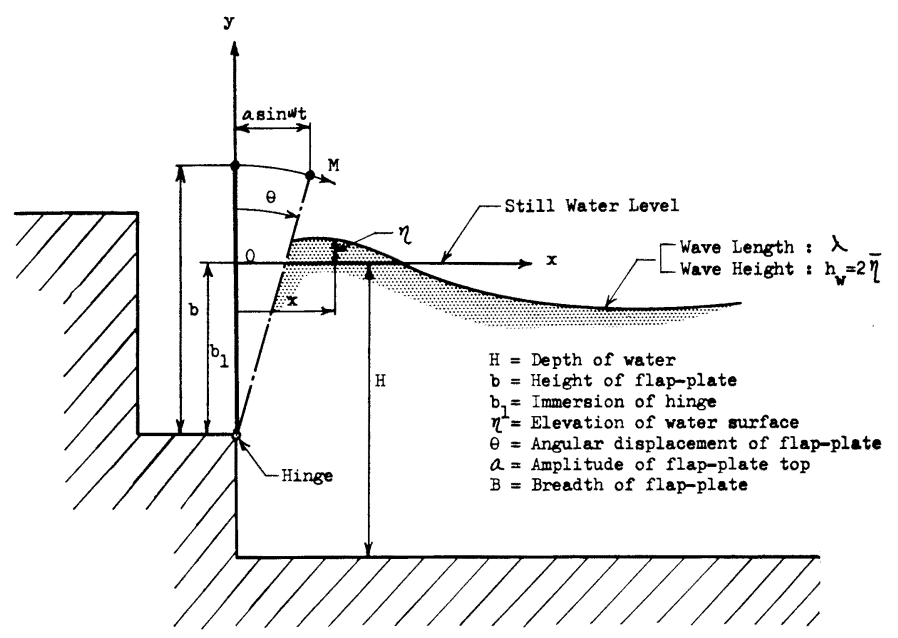

Fig. 1 Symbols and Coordinate Axes

and the other symbols and coordinate axes are taken as shown in Fig. 1 . If the water pressure on the flap is represented by $p$, the moment $M$ due to this water pressure about the hinge of the flap is represented by the following equation in the case of the wave maker without water on back side.

$$
M=\int_{-b_{1}}^{0} B p\left(b_{1}+y\right) d y
$$

and

$$
p=\rho\left(\frac{\partial \phi}{\partial t}\right)_{x=0}-\frac{1}{2} q^{2} \rho-\Omega \rho,
$$

where

$q=$ velocity of water particle

$\Omega=g y$ : gravity potential.

Substituting (5) into (4),

$$
\frac{M}{B \rho}=\int_{-b_{1}}^{0}\left(b_{1}+y\right)\left\{\left(\frac{\partial \phi}{\partial t}\right)_{x=0}-\frac{1}{2} q_{x=0}^{2}-\Omega\right\} d y .
$$

In the case of usual flap-type wave maker with water on both sides, the contributions of the 2 nd and 
the 3rd term in the bracket \{ \} of the right-hand side of (8) are cancelled on the both sides of the flap. So we have only to calculate the term $(\partial \phi / \partial t)_{x=0}$ in this case.

But in the case of the present type of wave maker without water on back side, we have to calculate all the terms of (8). Then,

$$
\left(\frac{\partial \phi}{\partial t}\right)_{x=0}=\frac{2 a \omega^{2}}{b}\left\{A \cosh k_{0}(y+H) \cos \omega t+\sin \omega t \sum_{m=1}^{\infty} B_{m} \cos k_{m}(y+H)\right\}
$$

and

$$
\begin{aligned}
q_{x=0}^{2} & =\left(\frac{\partial \dot{\phi}}{\partial x}\right)_{x=0}^{2}+\left(\frac{\partial \phi}{\partial y}\right)_{x=0}^{2} \\
\left(\frac{\partial \phi}{\partial x}\right)_{x=0} & =\frac{2 a \omega}{b}\left\{-A k_{0} \cosh k_{0}(y+H)+\sum_{m=1}^{\infty} k_{m} B_{m} \cos k_{m}(y+H)\right\} \cos \omega t \\
\left(\frac{\partial \phi}{\partial y}\right)_{x=0} & =\frac{2 a \omega}{b}\left\{A k_{0} \sinh k_{0}(y+H) \sin \omega t+\cos \omega t \sum_{m=1}^{\infty} k_{m} B_{m} \sin k_{m}(y+H)\right\},
\end{aligned}
$$

where

$$
\begin{aligned}
A & =\left\{\left(\frac{b_{1} \omega^{2}}{g}-1\right) \cosh k_{0} H+\cosh k_{0}\left(H-b_{1}\right)\right\} / k_{0} 3\left(H+\frac{g}{\omega^{2}} \sinh ^{2} k_{0} H\right) \\
B_{m} & =\left\{\left(\frac{b_{1} \omega^{2}}{g}-1\right) \cos k_{m} H+\cos k_{m}\left(H-b_{1}\right)\right\} / k_{m^{3}}\left(H-\frac{g}{\omega^{2}} \sin k_{m} H\right) .
\end{aligned}
$$

However, the calculation of the equation (10) is rather complicated because it involves the squares of the infinite series. So the following approximations were made considering the motion of the water particle near the flap.

$$
\begin{aligned}
& \left(\frac{\partial \phi}{\partial x}\right)_{x=0} \fallingdotseq \frac{\partial}{\partial t}\left\{\frac{a}{b}\left(b_{1}+y\right) \sin \omega t\right\}=\frac{a}{b}\left(b_{1}+y\right) \omega \cos \omega t \\
& \left(\frac{\partial \phi}{\partial y}\right)_{x=0} \fallingdotseq \frac{\partial}{\partial t}\left\{a \frac{b_{1}}{b} \operatorname{sech} \frac{\omega^{2} H}{g} \sinh \frac{\omega^{2}(H+y)}{g} \cos \omega t\right\}=-a \omega \frac{b_{1}}{b} \operatorname{sech} \frac{\omega^{2} H}{g} \sinh \frac{\omega^{2}(H+y)}{g} \sin \omega t
\end{aligned}
$$

Substituting $\left(11^{\prime}\right)$ and $\left(12^{\prime}\right)$ into (10),

$$
q_{x=0}^{2}=\left(\frac{a}{b}\right)^{2} \omega^{2}\left\{\left(b_{1}+y\right)^{2} \cos ^{2} \omega t+b_{1}^{2} \operatorname{sech}^{2} \frac{\omega^{2} H}{g} \sinh ^{2} \frac{\omega^{2}(H+y)}{g} \sin ^{2} \omega t\right\} .
$$

Substituting (9) and (15) into (8) and carrying out the integration,

$$
\begin{aligned}
\frac{M}{B \rho}= & \left(\frac{a}{b}\right) \omega^{2}\left\{2 A\left[\frac{b_{1}}{k_{0}} \sinh k_{0} H-\frac{1}{k_{0}^{2}}\left\{\cosh k_{0} H-\cosh k_{0}\left(H-b_{1}\right)\right\}\right] \cos \omega t\right. \\
& +2\left\{\sum_{m=1}^{\infty} B_{m}\left[\frac{b_{1}}{k_{m}} \sin k_{m} H-\frac{1}{k_{m^{2}}}\left\{\cos k_{m} H-\cos k_{m}\left(H-b_{1}\right)\right\}\right]\right\} \sin \omega t \\
& -\frac{1}{8}\left(\frac{a}{b}\right) b_{1}{ }^{4} \cos ^{2} \omega t+\frac{1}{6} \frac{g b_{1}^{3}}{(a / b) \omega^{2}} \\
& \left.+\frac{1}{8}\left(\frac{a}{b}\right) b_{1}{ }^{2} \operatorname{sech}^{2} \frac{\omega^{2} H}{g}\left[b_{1}^{2}-\frac{g b_{1}}{\omega^{2}} \sinh \frac{2 \omega^{2} H}{g}+\frac{1}{2}\left(\frac{g}{\omega}\right)^{2}\left\{\cosh \frac{2 \omega^{2} H}{g}-\cosh \frac{2 \omega^{2}\left(H-b_{1}\right)}{g}\right\}\right] \sin ^{2} \omega t\right)
\end{aligned}
$$

Further, the wave profile $\eta$ measured from the still water level may be taken

$$
\eta=\frac{1}{g}\left[\frac{\partial \phi}{\partial t}\right]_{y=0}
$$

under the assumption of small disturbance. Then substituting (1), (13) and (14) into (17),

$$
\eta=2\left(\frac{a}{b}\right) \frac{\omega^{2}}{g}\left\{A \cosh k_{0} H \cdot \cos \left(\omega t-k_{0} x\right)+\sin \omega t \sum_{m=1}^{\infty} B_{m} e^{-k_{m} x} \cos k_{m} H\right\} .
$$

Hence, if the dimensions of flap and basin and the motion of flap are given, the height of wave generated and the hydrodynamic moment to drive the flap are calculated by the equations (18) and (16), respectively. In Table 1 the wave height versus amplitude ratio and the components of driving force 
Table 1 An Example of Numerical Calculation

\begin{tabular}{c|c|c|c|c|c|c}
\hline & & \multicolumn{5}{|c|}{ Components of $F$ in the case $\lambda / h_{w}=20$} \\
\cline { 3 - 7 }$(\mathrm{m})$ & $\bar{\eta} / a$ & $\begin{array}{c}C \\
(\mathrm{~kg})\end{array}$ & $\begin{array}{c}\varphi \\
(\mathrm{rad})\end{array}$ & $\begin{array}{c}D \\
(\mathrm{~kg})\end{array}$ & $\begin{array}{c}E \\
(\mathrm{~kg})\end{array}$ & $\begin{array}{c}G \\
(\mathrm{~kg})\end{array}$ \\
\hline 0.5 & 1.8863 & 745.3 & 1.5582 & 0 & -0.86 & 3264.3 \\
1 & 1.7726 & 635.2 & 1.5153 & 0 & -1.72 & 3263.9 \\
2 & 1.5507 & 383.4 & 1.2433 & 0 & -3.41 & 3262.1 \\
3 & 1.3541 & 245.7 & 0.1654 & 0 & -4.87 & 3258.0 \\
4 & 1.1908 & 501.1 & -0.7131 & 0 & -5.71 & 3250.7 \\
5 & 1.0566 & 886.9 & -0.9353 & 0 & -5.46 & 3239.0 \\
6 & 0.9433 & 1280.6 & -1.0103 & 0 & -3.71 & 3221.8 \\
7 & 0.8459 & 1654.7 & -1.0382 & 0 & -0.11 & 3197.3 \\
8 & 0.7613 & 1995.2 & -1.0441 & -5.55 & 0 & 3169.6 \\
9 & 0.6876 & 2299.4 & -1.0377 & -13.22 & 0 & 3133.7 \\
10 & 0.6244 & 2568.1 & -1.0237 & -22.42 & 0 & 3088.2 \\
\hline
\end{tabular}

Note:

$F=\frac{M}{b_{1}}=C \cdot \cos (\omega t-\varphi)+D \cdot \sin ^{2} \omega t+E \cdot \cos ^{2} \omega t+G$.

Dimensions used; $H=3.5 \mathrm{~m}, \quad B=10 \mathrm{~m}, \quad b_{1}=1.4 \mathrm{~m}$

are shown for varying wave length as an example of a result of such numerical calculation. In this calculation the dimensions of flap and basin are taken the same as those of the new wave maker of the Basin, and $B_{m}$ is calculated up to $m=20$, at which the convergence of the infinite series was thought sufficient.

In order to design a driving mechanism, we have to know the total driving moment which is composed of a hydrodynamic one given by (16) and a moment balancing the inertia moment of the flap. tself. Let $M_{T}$ be the total driving moment and $M_{I}$ the moment balancing the inertia moment, then

$$
M_{T}=M+M_{I}
$$

and

$$
M_{I}=\frac{I}{g} \frac{d^{2} \theta}{d t^{2}}=-\frac{I}{g}\left(\frac{a}{b}\right) \omega^{2} \sin \omega t
$$

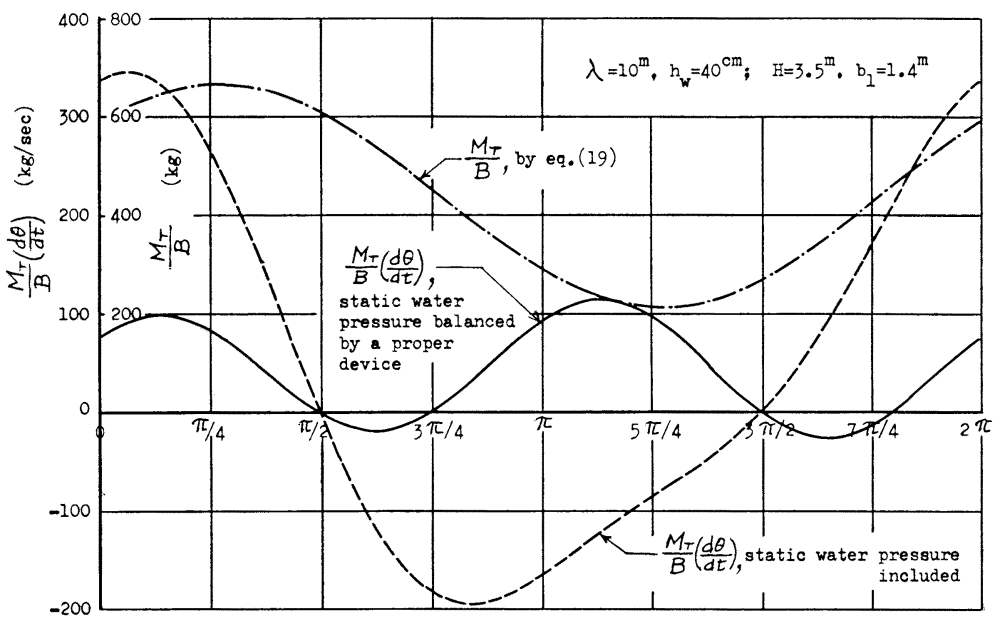

Fig. 2 Driving Moment and Power 
where $I$ is the moment of inertia of the flap about the hinge and $\theta$ is the angular displacement of the flap as shown in Fig. 1. As an example, Fig. 2 shows the driving moment and power $\left(M_{T} \times(d \theta / d t)\right)$ required to generate a wave of 40 centimeters in height and 10 meters in length in the Basin.

In the case of the present type of wave maker, however, we can economize in power substantially by a proper device, description of which is given in the following section.

\section{System Design and Treatment for Gaps between the Flap and Basin Wall}

As seen from the equation (16), the hydrodynamic driving moment consists of a varying part composed of four terms depending on $\sin \omega t, \cos \omega t, \sin ^{2} \omega t$ and $\cos ^{2} \omega t$, and a constant part due to the static water pressure. Hence, if the constant part of the driving moment is balanced by a suitable system such as a sufficiently long spring or a ram connected to a sufficiently large chamber filled with compressed air, the net driving moment can be reduced substantially as recognized from the data shown in Table 1. In this case the maximum power needed to drive the flap is about half of that in the case of usual flap-type wave maker with water on both sides.

In the practical design, the authors adopt an electro-hydraulic driving system, the details of which is described in Section 4. In this design the constant moment opposing the static water pressure is given by two hydraulic rams per each flap unit of 10 meters in breadth. The load delivered to the pistons is balanced by oil pressure which is kept nearly constant by means of an oil pressure circuit composed of an accumulator, a relief valve, a check valve and an oil pump driven by an electric motor. The oil pump supplies pressure oil only as much as relieved through the valve. Hence, the capacity of the oil pump can be fairly small. The varying part of moment opposing the net hydrodynamic load may be given by a usual electro-hydraulic servo system. The diagrammatic sketch of the system is shown in Fig. 6, in which the system for balancing the static water pressure is also in. cluded.

In the case of usual flap-type wave maker with water on both sides, there are two gaps between the flap ends and basin wall. Water on the back side goes out and comes in through these gaps in accordance with the motion of the flap and generates standing waves which disturb the formation of favourable waves. In the case of the present new type of wave maker, the gaps between the flap and the basin wall have to be water tight, because there is no water on the back side. Dr. Remmers2) developed a sliding type, in which small amount of water is allowed to leak at the sliding part and is used for cooling the oil pump. This type of wave maker seems to be suitable for a small basin. But, in the case of a large basin, the frictional power loss due to slide becomes very large and the leaking of water is not desirable for the maintenance of the large basin. The construction of the flap ends developed by the authors for the new flaptype wave maker without water on back side is shown in Fig. 3. As easily seen in the figure, the volume between the flap end and the basin wall is kept constant irrespective of the motion

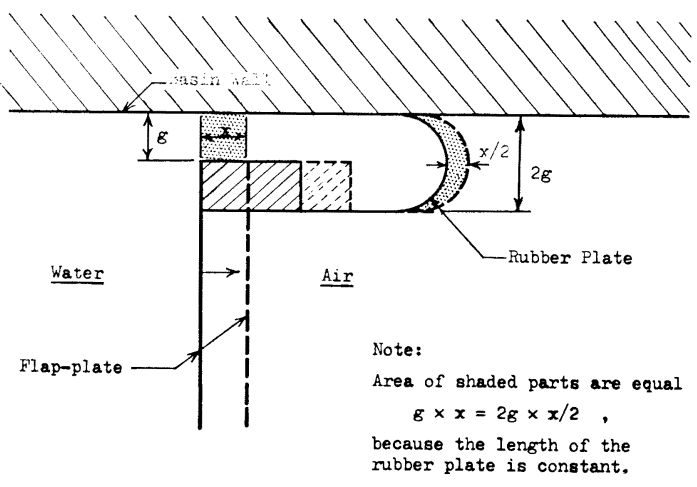

Fig. 3 Construction of Flap-plate End of the flap. Hence, the disturbance of water seen at the flap-end gaps of usual flap-type wave maker is completely eliminated and perfect water tightness can also be guaranteed by this construction. The lower edge of the flap is covered by a rubber plate. There may be no problem since the relative motion at this part of the flap is fairly small. 


\section{The Flap-type Wave Maker of the Seakeeping and Manoeuvring Basin}

The shape and dimensions of the Basin are shown in Fig. 4. The wave maker flaps are placed on the two adjacent sides of the Basin in order that the extensive seakeeping tests among oblique waves can be made by the use of the towing carriage capable of $x-y$ (length- \& side-wise) motion. In consideration of the outstanding merits discussed in the previous section, the flaps were arranged so as to make the back space of the flap completely free of water. The section of the flap and the back wall is shown in Fig. 5. The depth of the Basin was decided in order that the shallow water effect on the wave and the ship motion characteristics can entirely be avoided. The vertical position of the lower hinge of the flap was chosen so that the wave generating surface of the flap fits the attenuating exponent of the orbital motion of the water particles in waves.

The flap of the wave maker is driven by an electro-hydraulic servo control system. The block diagram of the actuation control is shown in Fig. 6. The longer side wave maker consists of twelve flap units actuated by twelve hydraulic rams, and the shorter one three flap units actuated by three rams, each flap unit being 10 meters in breadth. The twelve flap units of the longer side and three flap units of the shorter side are connected water-tightly each other by rubber sheet so that they can

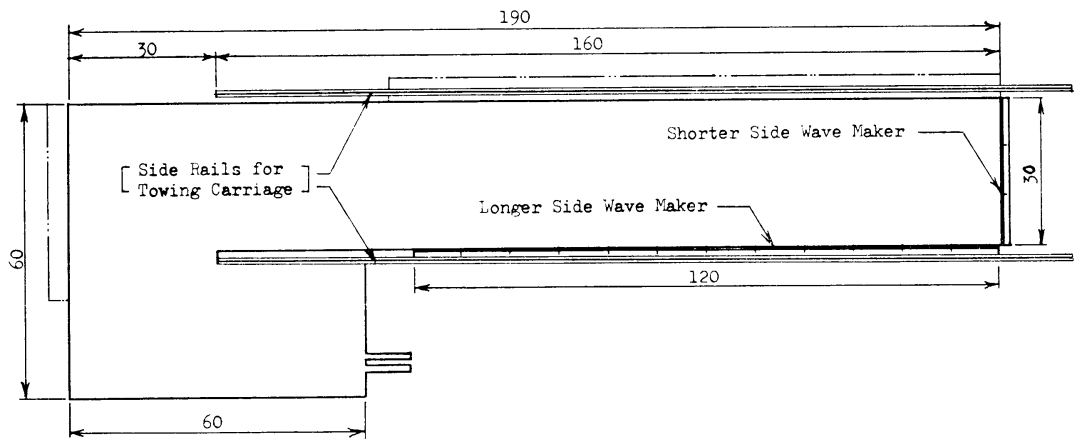

Fig. 4 Seakeeping and Manoeuvring Basin

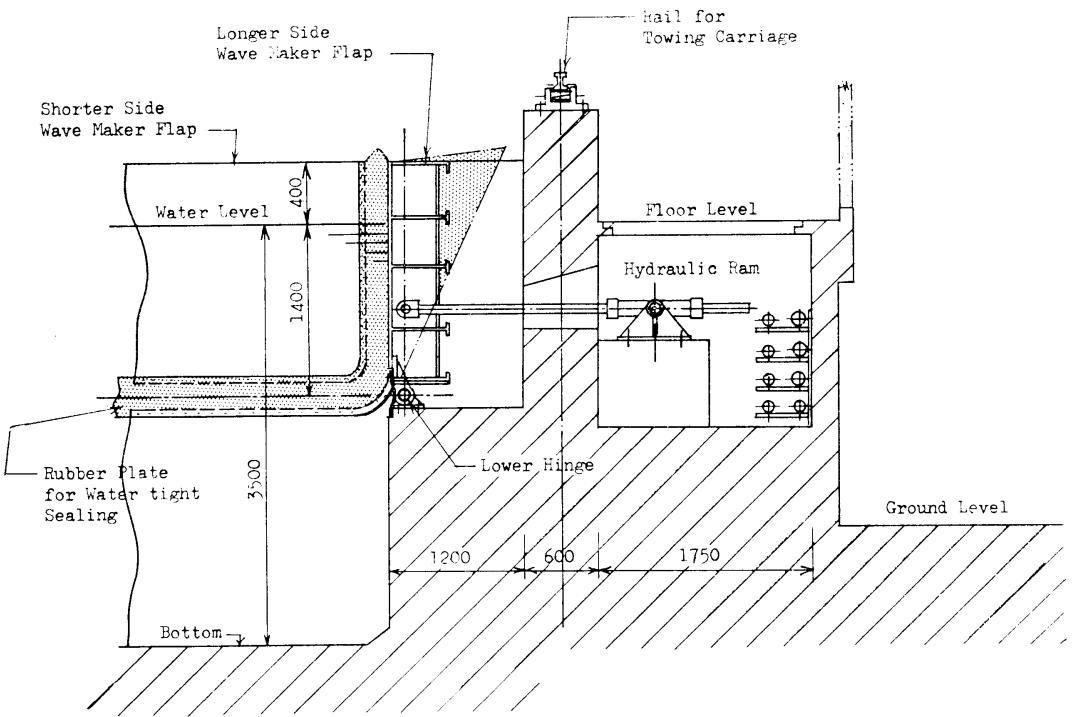

Fig. 5 Section of Flap and Wall 


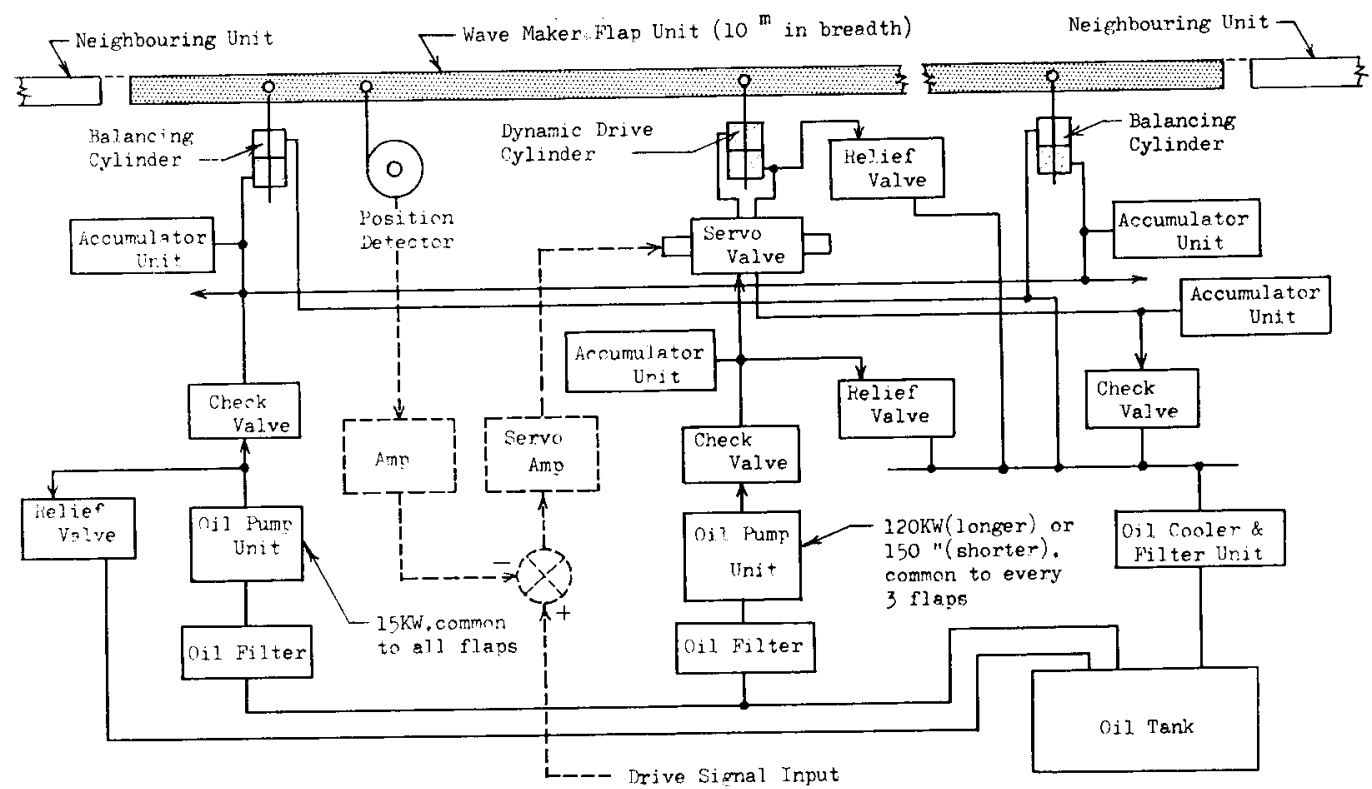

Fig. 6 Electro-hydraulic Servo System

oscillate as a single flap of 120 meters and 30 meters in breadth, respetively. The so-called snake-type motion of flaps is not intended in the Basin. Extensive design studies have been made on the response characteristics of the servo control system by the use of the simulated model plant with very long oil piping arrangement together with digital and analog computer simulation techniques.

Design studies have also been made on the structural characteristics of the flap. In the course of the studies, theoretical calculations for the deflection and the vibration characteristics were confirmed by the careful model tests carried out by the use of the $1 / 10$ scale structural model. The deflection of the flap under the designed maximum load is limited within $1 \%$ of the amplitude of the flap.

As described in the previous section, the water tight sealers at the flap ends and the bottom edge of the flap are made of flexible rubber plate specially chosen and prepared in consideration of the repeated deflections. Tests were made to certify the durability of the rubber plate against the frequent change of the bending and the membrane tension due to hydrodynamic pressure up to $10^{8}$ times or more.

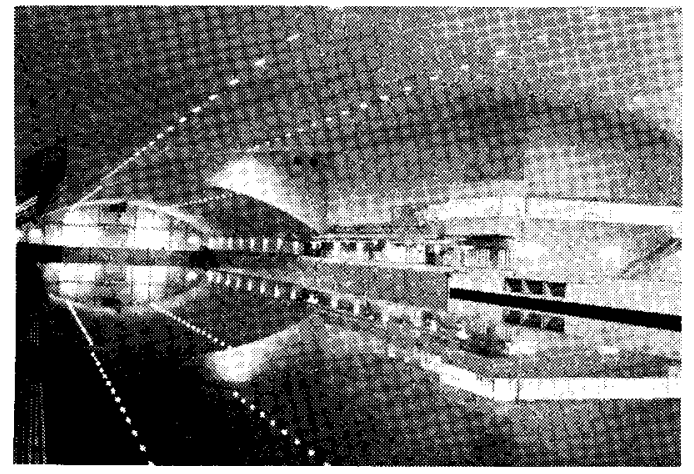

Fig. 7 Wave Maker Flap Arrangement and Control Room

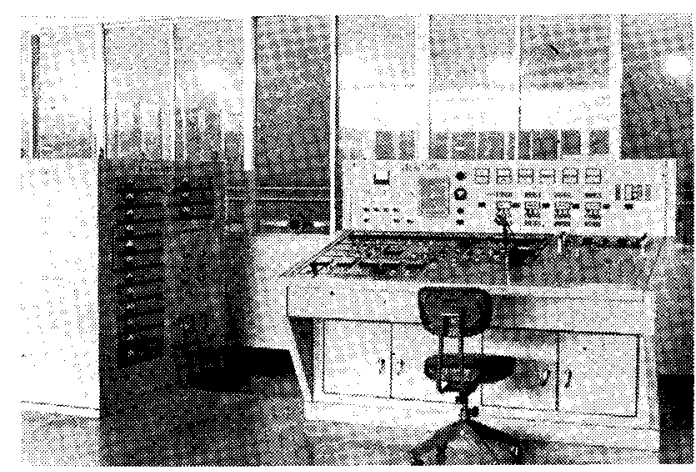

Fig. 8 Wave Maker Control Station 
The full controls of the wave maker system can be made at the control room looking over the Basin. In Figs. 7 and 8 are shown the photographs of the wave maker flap arrangement and the control station.

This new type wave maker of the Basin is now under the trial run and proving the characteristics expected. The details of the trial results will be reported in the next chance.

\section{Acknowledgement}

The basic development of this new flap-type wave maker without water on back side was carried out by the present authors. However, the building of the whole system was completed by the cooreration of many participants under the supervision of the authors.

The manufacturing design and the building business were carried out by Mr. K. Harada of Nagasaki Shipbuilding and Engine Works, M.H.I., the basic design of the electro-hydraulic servo system and the analysis of its dynamical characteristics was made by Mr. S. Mitsumori of Nagasaki Technical Institute, the electro-hydraulic drive and control system was built by Messrs. Tokyo Keiki Co., Ltd. and the flap-plates were built by Messrs. Kyuki Co., Ltd.

The authors wish to thank all these participants and the staff of Nagasaki Technical Institute for their cooperation.

\section{References}

1) Taniguchi, K. \& Shibata, J.: "On the wave maker of Mitsbuishi Nagasaki Experimental Tank." Journal of the Soc. Naval Arch. of West Japan, No. 9 (May 1955), (in Japanese).

2) Messrs. Kempf \& Remmers: Description of Wave Making Machine W 23. 